\title{
Numerical Simulation of The Response of An Underground Pipeline Connector With a Super-Large Section Under Complex Geological Conditions
}

Long Ma ( $\sim$ 49186553@qq.com )

Hohai University

Ping Ai

Hohai University

ChuanSheng Xiong

Hohai University

\section{Research Article}

Keywords: Complex geological conditions, Super-large section, Underground integrated pipe gallery joint, Response numerical simulation

Posted Date: October 21st, 2021

DOl: https://doi.org/10.21203/rs.3.rs-970736/v1

License: (c) (i) This work is licensed under a Creative Commons Attribution 4.0 International License. Read Full License 


\title{
Numerical simulation of the response of an underground pipeline connector with a super-large section under complex geological
} conditions

\author{
Long Ma ${ }^{1, *}$, Ping Ai ${ }^{2}$, ChuanSheng Xiong ${ }^{3}$ \\ ${ }^{1}$ College of Computer and Information Engineering,Hohai University,Nanjing 211100,China \\ ${ }^{2}$ College of Computer and Information Engineering,College of Hydrology and Water Resources,Hohai \\ University,Nanjing 210098,China \\ ${ }^{3}$ College of Hydrology and Water Resources,Hohai University,Nanjing 210098,China \\ *49186553@qq.com
}

\begin{abstract}
Aiming at the low simulation accuracy of the numerical simulation method of the joint response of the super-large section underground comprehensive pipeline gallery under the current complicated geological conditions, a numerical simulation method of the joint response of the super-large section underground comprehensive pipe gallery joint response under the complicated geological conditions based on the finite element model was proposed. According to the analysis process for the super-large section underground comprehensive pipe gallery, the viscous boundary of the comprehensive pipe gallery is determined. Additionally, by analyzing soil and structural parameters, the optimal combined dynamic boundary is used as the model boundary. The HSS model is used to describe the constitutive structure of the soil, and by improving the Goodman element to describe the contact surface of the model, the finite element model of the joint response of the comprehensive pipe gallery is constructed. Furthermore, based on the internal force balance and deformation coordination conditions, considering the influence of the deformation shape of the joint joints and the elongation of the prestressed tendons on the finite element model, the response model of the integrated pipe gallery joint is optimized. Experimental results show that the proposed method has higher numerical simulation accuracy.
\end{abstract}

Key words: Complex geological conditions; Super-large section; Underground integrated pipe gallery joint; Response numerical simulation

\section{Introduction}

The underground integrated pipe gallery is a modern and intensive urban infrastructure formed when two or more urban pipelines are centrally set up in the same artificial space. Because this infrastructure has more advantages than directly buried pipelines, it can be anticipated as an inevitable trend in the development of urban modernization. The complex geological conditions in my country and the existence of super-large cross-sections make the study of the joint response of the super-large cross-section underground comprehensive pipeline gallery under complex geological conditions important 1,2 . The pipeline can withstand long-term and short-term loads related to the influence of various external factors, such as the movement of surrounding soil masses, accidental mechanical shock, operation of auxiliary equipment valves, movement of online inspection equipment, and changes in gas transportation methods 3,4 . 
The dynamic influence on the pipeline is one of the most important factors leading to pipeline damage. It may be caused by seismic events, fault movement, or soil displacement in the pipeline area. In many cases, the work of construction equipment near the pipeline generates dynamic loads. This problem is particularly common for ultra-large cross-section underground pipeline connectors located in complex geological conditions ${ }^{5,6}$.

At the present time, many researchers are very interested in developing automated systems for monitoring the mechanical state of such objects. The possibility that natural gas pipelines in operation have various mechanical characteristics has been studied. The main reason for the vibration of pipeline parameters, particularly the micro-seismic pipeline vibration related to pipeline operation, consider the compression station ${ }^{7}$. The analysis and numerical study of pipeline vibration caused by valve opening has been conducted ${ }^{8,9}$. Bochkarev et al. ${ }^{10}$ studied the vibration caused by the movement of objects in the pipeline. Stajanca et al. ${ }^{11}$ studied the impact of pipeline vibration response on gas leakage. Different methods exist for conducting experimental research on the mechanical conditions of gas pipelines: vibration sensors ${ }^{12,13}$; strain gauge applications ${ }^{14}$; checking the capabilities of optical fiber sensors ${ }^{15}$, 16 ; and acoustic and ultrasonic detection methods ${ }^{17,18}$.

The indoor test cost is high and the cycle is long. Numerical simulation has become an important means for the joint response analysis of the super-large section underground comprehensive pipeline gallery ${ }^{19}$. For such analysis under complex geological conditions, the current widely used numerical simulation technology is to cut a finite-size near-field calculation area from the super-large section under complex geological conditions. At this time, it is necessary to establish a virtual artificial boundary on the boundary of the computational domain to eliminate reflection and simulate the radiation damping effect of the far-field medium ${ }^{20}$.

Under complex geological conditions, the joint response of the super-large section underground comprehensive pipeline gallery has attracted increasingly relevant experts and scholars. Some mature theories and applications have been produced in this field. However, these methods still have some shortcomings that need to be studied and resolved. For example, literature ${ }^{21}$ proposed a method based on finite element analysis to study the dynamic response of the underground pipe gallery. In the study, ANSYS/LS-DYNA dynamic finite element analysis software was used. Based on the fluid-solid coupling algorithm, the process of gas explosion propagation inside the underground pipe gallery structure was numerically simulated. The shock wave in the explosion process, the propagation process, and the pressure 
cloud diagram of the shock wave were obtained, and the dynamic response law of the pipe gallery structure was analyzed. However, this method did not fully excavate the laws of the dynamic response of the geological conditions to the underground pipe gallery, which resulted in the low accuracy of the numerical simulation. Literature ${ }^{22}$ proposed a numerical analysis method for the influence of the soil-structure interface parameters of the underground integrated pipeline gallery on the seismic dynamic response. Through the establishment of a dynamic finite element numerical model, the effects of different ground motion inputs and changes in contact surface reduction coefficients on the internal force and acceleration of the integrated pipe gallery were studied, respectively. However, this method did not result from in-depth research on the influencing factors of integrated pipe gallery joints, which affected the accuracy of the numerical simulation. Literature ${ }^{23}$ proposed a method to study the fire smoke temperature field of the urban underground comprehensive pipe gallery. A 1:3.6 small-scale comprehensive pipe gallery model was established. The researchers changed the fire source power by changing the size of the gasoline pan, and an oil pool fire experiment was conducted. Origin software was used to analyze the temperature data. The empirical formula for the attenuation of the flue gas temperature and distance was obtained. The fire smoke temperature field of the urban underground comprehensive pipe gallery was studied. However, in the application process of this method, the materials of the integrated pipe gallery were not analyzed, which affected the accuracy of the numerical simulation. Literature ${ }^{24}$ proposed a numerical simulation method based on the regression equation for the response of integrated pipe gallery joints. By analyzing the transmission process of the comprehensive pipe gallery, the researchers constructed the relationship function between the design factors and the dynamic index, and improved the accuracy of the relationship model using the regression equation. According to the relational model, the joint response of the integrated pipe gallery was numerically simulated. However, in the application process of this method, the factors that affected the response of the integrated pipe gallery joints were not completely extracted, which resulted in the low accuracy of the numerical simulation.

To solve the problems of the above algorithm, a numerical simulation method based on the finite element model of the joint response of the super-large section underground comprehensive pipeline gallery under complex geological conditions is proposed. The overall structure is as follows:

1) Through the viscous boundary of the comprehensive pipe gallery, the finite element model of the joint response of the super-large section underground comprehensive pipe gallery under complex geological conditions is constructed using the analysis of the soil and structural parameters. 
2) Based on the internal force balance and deformation coordination conditions, considering the influence of the joint deformation shape and prestressed tendon elongation on the finite element model, the integrated pipe gallery joint response model is optimized.

3) The experimental data demonstrate the feasibility and effectiveness of the numerical simulation method based on the finite element model of the super-large section underground comprehensive pipeline gallery joint response under complex geological conditions.

4) Based on the research in this paper, a prospect is proposed.

\section{Numerical simulation of the joint response of the super-large section underground comprehensive pipe gallery under complex geological conditions}

\section{Modeling of the joints of the super-large cross-section underground comprehensive pipeline gallery under complex geological conditions}

To model the joints of the super-large section underground comprehensive pipe gallery under complex geological conditions, it is necessary to analyze the pipe gallery under such conditions. The entire analysis is conducted in three steps: the first step is static analysis. Under complex geological conditions, the conditions required for the super-large section soil to reach the balance of initial stress and pore water pressure are determined. In the second step, while the soil is being excavated, the underground comprehensive pipe gallery structure is added. Calculations are performed to achieve the stress and pore water pressure balance. The third step is dynamic analysis. The stress time history is input at the bottom of the model to dynamically calculate the interaction between the integrated pipe gallery and the soil. In the dynamic analysis process, a dynamic constitutive model that can consider the change of excess pore water pressure of soil liquefaction is used to reflect the influence of the super-large section on the joints of the underground comprehensive pipe gallery under complex geological conditions. The geometric dimensions of the cross-section of a single warehouse of the underground integrated pipe gallery are shown in Figure 1. 


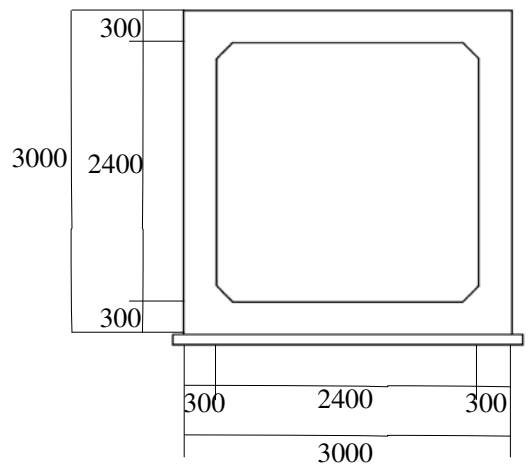

Figure 1. Geometry size of a single warehouse section of the underground comprehensive pipe gallery

The basic idea of the viscous boundary is to set a damper on the artificial boundary to absorb the energy of the scattered wave in the vibration process. PLAXIS software provides viscous boundary conditions based on this method. In the joint analysis of the integrated pipe gallery, if a viscous boundary is set at the bottom of the model, the transmission process of the integrated pipe gallery should be the load time history.

Consider a damper in the $x$ direction on the boundary as an example. The absorbed normal and tangential stress components can be expressed as

$$
\left\{\begin{array}{l}
\sigma_{N}=-C_{1} \rho v_{P} \dot{u}_{x} \\
\tau=-C_{2} \rho v_{S} \dot{u}_{y}
\end{array},\right.
$$

where $\sigma_{N}$ is the normal stress acting along the viscous boundary. $\tau$ is the tangential stress acting along the viscous boundary. $\dot{u}_{x}$ is the moving speed of the particle normal to the boundary. $\dot{u}_{y}$ is the velocity of the particle tangential to the boundary. $\rho$ is the density of the medium. $v_{P}$ and $v_{S}$ are the compression wave velocity and shear wave velocity, respectively. $C_{1}$ and $C_{2}$ are relaxation coefficients.

The relaxation coefficients $C_{1}$ and $C_{2}$ are used to improve the wave absorption effect of the viscous boundary: $C_{1}$ adjusts the energy dissipation along the normal direction of the boundary and $C_{2}$ adjusts the energy dissipation along the tangential direction of the boundary. If the boundary is only subject to normal fluctuations, there is no need to consider relaxation; at this time $C_{1}=C_{2}=1$. Generally, the fluctuation direction is not only perpendicular to the boundary but also along any direction. At this time, the value of $C_{2}$ should be adjusted to improve the absorption effect. 
The viscous boundary and viscoelastic boundary are more effective for internal problems, such as blasting. However, because they belong to the absorption boundary, the main consideration is the absorption effect of the external traveling wave. The transmission problem of the integrated pipe gallery joint is not considered, and the joint problem of the integrated pipe gallery cannot be well analyzed. Typically, the joint of the integrated pipe gallery is considered as the bottom boundary of the vertical incidence, and the traveling wave field inside the two sides of the boundary is a free wave field at this time. The free field boundary passes through the "forced" free field movement while ensuring that the outgoing waves generated in the engineering calculation area are properly absorbed to simulate the boundary conditions of the infinite domain. The finite element software obtains the free-field boundary conditions by generating free-field grids corresponding to the boundary nodes around the model. The side boundary of the main grid and the free field grid are coupled through a damper. In the solution process, the free field grid also performs synchronous calculations when the main grid is calculated, and the unbalanced force of the free field grid is applied to the side boundary of the main grid to meet the displacement and stress conditions on the side boundary. The conditions provided by the free field are exactly the same as those provided by the infinite field. The expressions of the unbalanced forces $F_{x}, F_{y}$, and $F_{z}$ along the $x$, $y$, and $z$ directions, respectively, applied on the free field side contact surface are:

$$
\left\{\begin{array}{l}
F_{x}=-\rho v_{P}\left(v_{x}^{m}-v_{x}^{f f}\right) A+F_{x}^{f f} \\
F_{y}=-\rho v_{S}\left(v_{y}^{m}-v_{y}^{f f}\right) A+F_{y}^{f f} \\
F_{z}=-\rho v_{S}\left(v_{z}^{m}-v_{z}^{f f}\right) A+F_{z}^{f f}
\end{array}\right.
$$

where $A$ denotes the affected area of the free field node; $v_{x}^{m}, v_{y}^{m}$, and $v_{z}^{m}$ are the velocity of the boundary nodes of the main grid along the $x, y$, and $z$ directions, respectively. $v_{x}^{f f}, v_{y}^{f f}$, and $v_{z}^{f f}$ are the speed of the free field node along the $x, y$, and $z$ directions, respectively. $F_{x}^{f f}, F_{y}^{f f}$, and $F_{z}^{f f}$ are the nodal forces generated by the normal stress in the $x, y$, and $z$ directions of the free field element, respectively.

The soil material structure adopts ABAQUS's own Drucker-Prager model ${ }^{25}$. The structure adopts linear elastic materials, and the model parameters are shown in Table 1:

$$
[C]=\alpha[M]+\beta[K],
$$

where 


$$
\begin{gathered}
\alpha=2\left(\frac{\xi_{1}}{\omega_{1}}-\frac{\xi_{2}}{\omega_{2}}\right) /\left(\frac{1}{\omega_{2}^{2}}-\frac{1}{\omega_{1}^{2}}\right) \\
\beta=2\left(\xi_{2} \omega_{2}-\xi_{1} \omega_{1}\right) /\left(\omega_{2}^{2}-\omega_{1}^{2}\right),
\end{gathered}
$$

where $\xi_{1}$ and $\xi_{2}$ are the damping ratios of the first and second modes, respectively. $\omega_{1}$ is the first-order frequency. $\omega_{2}$ depends on the input integrated pipe gallery joint, and its value should ensure that it can cover most of the frequency information of the integrated pipe gallery joint.

Table 1 Soil and structural parameters in the model

\begin{tabular}{ccc}
\hline Parameter & Soil & Structure \\
\hline Density $/\left(\mathrm{kg} * \mathrm{~m}^{3}\right)$ & 1800 & 2400 \\
Elastic Modulus $/(\mathrm{MPa})$ & 10 & 33000 \\
Poisson's ratio/\% & 40 & 20 \\
Internal friction angle $/\left(^{\circ}\right)$ & 27.9 & $/$ \\
Cohesion $/ \mathrm{kPa}$ & 24.4 & $/$ \\
Dilatancy angle $/\left({ }^{\circ}\right)$ & 0 & $/$ \\
\hline
\end{tabular}

Considering the triangular elements in the two-dimensional grid as an example, the relationship between the contact surface elements and the soil elements required to model the joints of the super-large cross-section underground comprehensive pipeline gallery under the complicated geological conditions is shown in Figure 2. The attributes of the contact surface elements in the super-large-section underground comprehensive pipe gallery are related to the model parameters of the surrounding soil. These parameters depend on the material model selected for the surrounding soil. The strength of the contact surface is defined by the parameter $R_{\text {inter }}$. Because the contact surface of soil structure interaction is generally lower than the strength of adjacent soil layers, $R_{\text {inter }}$ should be less than 1 . If the contact surface is elastic, not only sliding (relative movement parallel to the contact surface) but also spreading or overlapping (e.g., relative displacement perpendicular to the contact surface) may occur. 


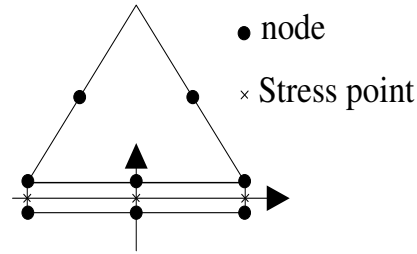

(a) 6 nodes

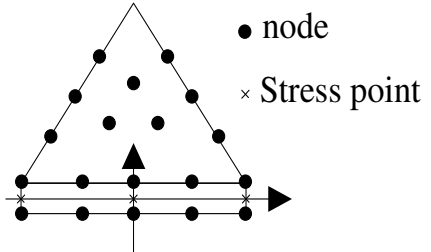

(b) 15 nodes

Figure 2. Distribution of nodes and stress points in the contact surface element and its connection with the soil element

According to the above, the elastic opening displacement and elastic sliding displacement of the joints of the super-large section underground comprehensive pipe gallery under complex geological conditions can be expressed as

$$
\begin{gathered}
w_{n}=\frac{\sigma}{K_{n}}=\frac{\sigma t_{i}}{E_{o c d, i}}, \\
w_{s}=\frac{\sigma}{K_{s}}=\frac{\tau^{\prime} t_{i}}{G_{i}},
\end{gathered}
$$

where $G_{i}$ is the shear modulus of the contact surface. $E_{o c d, i}$ is the one-dimensional compressive modulus of the contact surface. $t_{i}$ is the virtual thickness of the contact surface. $K_{n}$ is the axial stiffness of the elastic contact surface. $K_{s}$ is the shear stiffness of the elastic contact surface.

According to the relationship between the shear modulus and compression modulus, the reduction factor $R_{\text {inter }}$ reduces not only the strength of the contact surface but also its stiffness. The key factor that affects the stiffness of the contact surface is its virtual thickness. The program automatically determines the virtual thickness to ensure that the contact surface has sufficient rigidity. When the contact surface strength reaches its limit value determined by $R_{\text {inter }}$, the contact surface strength can be softened to a reduced value determined by $R_{\text {inter }}$.

When the shear stress $\tau$ satisfies the following formula, the contact surface remains elastic:

$$
|\tau|<-\sigma_{n} \tan \varphi_{i}+c_{i}
$$

When the shear stress $\tau$ satisfies the following formula, the contact surface exhibits plastic behavior:

$$
|\tau|=-\sigma_{n} \tan \varphi_{i}+c_{i}
$$


where $\varphi_{i}$ and $c_{i}$ are the friction angle and the cohesion force of the contact surface, respectively.

The strength properties of the contact surface are related to those of the rock and soil layers. Each material data group contains a corresponding $R_{\text {inter }}$, which is calculated according to the following rules:

$$
\begin{gathered}
c_{i}=R_{\mathrm{inter}} \sigma_{n}, \\
\tan \varphi_{i}=R_{\mathrm{inter}} G_{i}+E_{\text {oed }, i} .
\end{gathered}
$$

In addition to the Coulomb shear stress criterion, the contact surface also follows the tensile truncation criterion:

$$
\sigma_{n}<R_{\mathrm{inter}} \sigma_{t, \text { soil }}
$$

where $\sigma_{t, \text { soil }}$ represents the tensile strength of the soil. When the tensile strength of the contact surface is reached, cracks (i.e., openings) may appear between the structure and the soil. In the subsequent reverse loading, the compressive stress can only continue to be generated after contact between the structure and the soil body is restored. The simulation of this process can be realized in PLAXIS. According to the importance of $R_{\text {inter }}$, the joint model of the super-large section underground comprehensive pipeline gallery under complex geological conditions is established to improve the numerical simulation accuracy of the joint response of the pipeline gallery under such conditions.

\section{Numerical simulation of the joint response}

In the numerical simulation of the joint response of the super-large section underground comprehensive pipe gallery under complex geological conditions, the joint test specimen in Fig. 3 is used as a prototype.

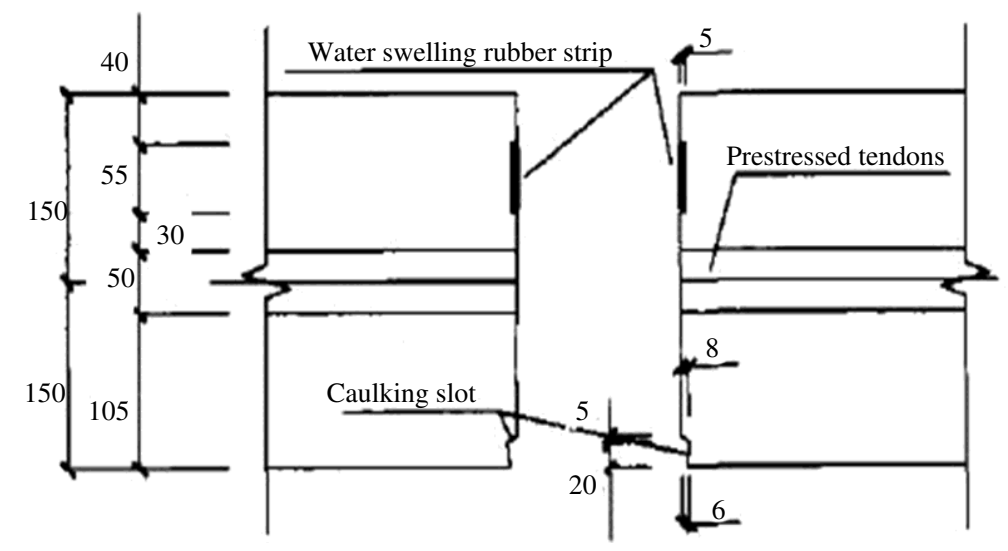

Figure 3. Joint test specimen 
The analysis according to 2.1 shows that the deformation of the joint test piece of the precast prestressed comprehensive pipe gallery is mainly caused by the relative rotation of the precast concrete slab. The tensile length of the prestressed tendons and the short compression of the concrete in the compression zone of the joint are the main reasons for the relative rotation of the precast concrete slab. The elongation of the prestressed tendons can be calculated by equation (13). Under the action of an external load, the stress increment of the prestressed tendons at the joint is A. The stress increment transfer length of the prestressed tendons is L. As shown in Figure 4, the stress transfer length and bond stress distribution form can be determined according to 6.1.9 and Article 8.1.8 of the "Code for Design of Concrete Structures" (GB50010-2002) ${ }^{26}$, as in equation (14):

$$
\begin{gathered}
\Delta \delta_{p}=\int_{0}^{l_{t r}} \Delta \varepsilon_{p z} d z=\frac{1}{2} \Delta \varepsilon_{p z} l_{t r}, \\
l_{t r}=\lambda \frac{\Delta \sigma_{p z}}{f_{t k}} d,
\end{gathered}
$$

where $\Delta \delta_{p}, \varepsilon_{p z}$, and $\Delta \sigma_{p z}$ are the incremental deformation, incremental strain, and incremental stress of the prestressed tendons at the joint joints relative to the decompression state. $l_{t r}$ is the transfer length of the stress increment of the prestressed tendons. $\lambda$ is the shape factor of the prestressed tendons. $d$ is the diameter of the prestressed tendons. $f_{t k}$ is the standard value of concrete tensile strength.

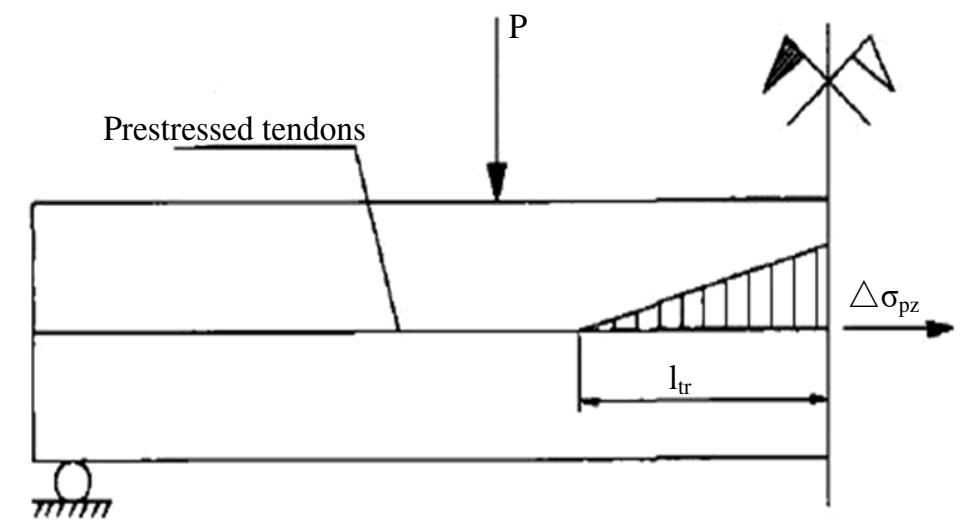

Figure 4. Stress transfer length of the prestressed tendons

It can be seen from the above that after the joints are opened, the joints of the prefabricated prestressed comprehensive pipe gallery meet the assumption of the flat section in the joint contact area, the breakaway area, and the entire section height range.

The constitutive relationship for the prestressed tendons adopts an ideal elastoplastic constitutive model, and constitutive relationship for concrete adopts the Hognestad constitutive model, defined as 
follows:

Prestressed tendons:

$$
\left\{\begin{array}{l}
\sigma_{p}=\varepsilon_{p} E_{p}, \varepsilon_{p} \leq \varepsilon_{p y} \\
\sigma_{p}=\varepsilon_{p y}, \quad \varepsilon_{p} \leq \varepsilon_{p y}
\end{array} .\right.
$$

Concrete:

$$
\left\{\begin{array}{l}
\sigma_{c}=f_{c}^{\prime}\left[\frac{2 \varepsilon_{c}}{\varepsilon_{0}}-\left(\frac{\varepsilon_{c}}{\varepsilon_{0}}\right)^{2}\right], \varepsilon_{c} \leq \varepsilon_{0} \\
\sigma_{c}=f_{c}^{\prime}\left[1-0.15 \frac{\varepsilon_{c}-\varepsilon_{0}}{\varepsilon_{\max }-\varepsilon_{0}}\right], \varepsilon_{c}>\varepsilon_{0}
\end{array}\right.
$$

From the assumption of the flat section of the joint,

$$
\begin{gathered}
\frac{\varepsilon_{c}}{y_{1}}=\frac{\varepsilon_{c e}}{y_{2}}, \\
y_{1}+y_{2}=y, \\
\frac{\varepsilon_{c}}{y_{1}}=\frac{\Delta \varepsilon_{p z}}{\left|\frac{h}{2}-y_{1}\right|}, \\
\frac{\Delta \varepsilon_{p}}{\left|\frac{h}{2}-y\right|}=\theta,
\end{gathered}
$$

where $h$ is the height of the joint section. $y_{1}, y_{2}$, and $y$ are the height of the concrete section in the nip, height of the concrete section in the tensioning zone, and total height of the joint surface contact zone generated by the incremental bending moment in the joint contact zone, respectively. $\varepsilon_{c e}, \varepsilon_{c}$, and $\theta$ are the pre-compression strain of the concrete when the prestress is applied, concrete strain at the outer edge of the compression zone of the joint surface, and joint surface rotation angle generated under the action of the bending moment load, respectively.

The constitutive structure of the prestressed tendons is substituted into equation (13), and then

$$
\Delta \delta_{p}=\frac{\lambda E_{p} d}{2 f_{t k}} \Delta \varepsilon_{p z}^{2},
$$

where $E_{p}$ is the elastic modulus of the prestressed tendons. $d$ is the diameter of the prestressed 
tendons.

Equation (21) is substituted into the assumption of the flat section of the joint to obtain

$$
\begin{gathered}
\varepsilon_{c}=\sqrt{\frac{2 f_{t k} \theta y_{1}^{2}\left|\frac{h}{2}-y\right|}{\lambda E_{p} d\left(\frac{h}{2}-y_{1}\right)^{2}},} \\
y_{2}=\frac{\sigma_{c e}}{E_{c}} \sqrt{\frac{\lambda E_{p} d\left(\frac{h}{2}-y_{1}\right)^{2}}{2 f_{t k} \theta y_{1}^{2}\left|\frac{h}{2}-y\right|}},
\end{gathered}
$$

where $E_{c}$ is the elastic modulus of concrete. $\sigma_{c e}$ is the pre-compression stress of concrete when the prestress is applied.

The failure morphology of the joints of the super-large section underground comprehensive pipe gallery under complex geological conditions shows that the concrete in the compression zone of the joints of the underground comprehensive pipe gallery is crushed and the prestressed tendons yield at the ultimate failure. The failure mechanism of the underground integrated pipe gallery joint is the truss mechanism. From the internal force balance of the joint splicing,

$$
y_{c}=\sum \frac{\varepsilon_{c}}{y_{1}}+\frac{f_{p y} b A_{p}}{f_{c}},
$$

where $f$ is the yield stress of the prestressed tendons. A is the cross-sectional area of the prestressed tendons. $\mathrm{f}$ is the compressive strength of concrete. $\mathrm{b}$ is the cross-sectional width of the joint. $\mathrm{y}$ is the height of concrete in the compression zone of the joint.

Then the ultimate compressive bearing capacity of the joint of the super-large section underground comprehensive pipe gallery under complex geological conditions is

$$
M_{u}=\frac{f_{p y} A_{p}}{y_{c}}\left(\frac{h}{2}-E_{c}+y_{2}\right) .
$$

Through the above discussion, the ultimate compressive bearing capacity of the joints of the super-large cross-section underground comprehensive pipe gallery under complicated geological conditions is used to numerically simulate the joints of the underground comprehensive pipe gallery.

\section{Experimental results and analysis}


To verify the validity and feasibility of applying the numerical simulation method based on the finite element model to the response of the super-large section underground comprehensive pipe gallery joint under complex geological conditions, an experiment was conducted. The experimental results are as follows.

To simulate the Rayleigh wave generated by the dynamic load, a pulse excitation load was applied to the boundary based on the method for applying the load in the Lamb (1904) problem. The dynamic load was simulated by a load that changed in a triangle with time. The load was applied after $0.05 \mathrm{~s}$, the duration of the load was $0.025 \mathrm{~s}$, and the amplitude of the load was $50 \mathrm{kN}$.

The bottom dynamic time history adopts the Upland seismic wave of California, USA. The peak acceleration was $0.2 \mathrm{~g}$ (Figure 5). Its intensity was moderate, which matches the intensity of Rayleigh waves. After a trial calculation, under the action of this seismic wave, the simultaneously activated Rayleigh wave can have a significant impact.

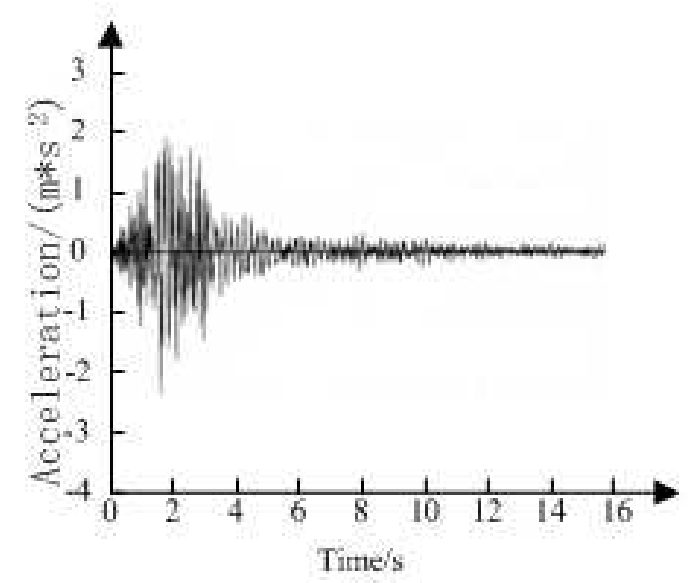

Figure 5. Acceleration time curve of the Upland, California seismic wave

The acceleration magnification coefficients of $0.1 \mathrm{~g}, 0.4 \mathrm{~g}$, and $1.0 \mathrm{~g}$ input in the lateral non-uniform excitation test of the joints were compared, and the results obtained are shown in Figure 6. The magnification factor is a constant, and its unit is $\mathrm{o}$.

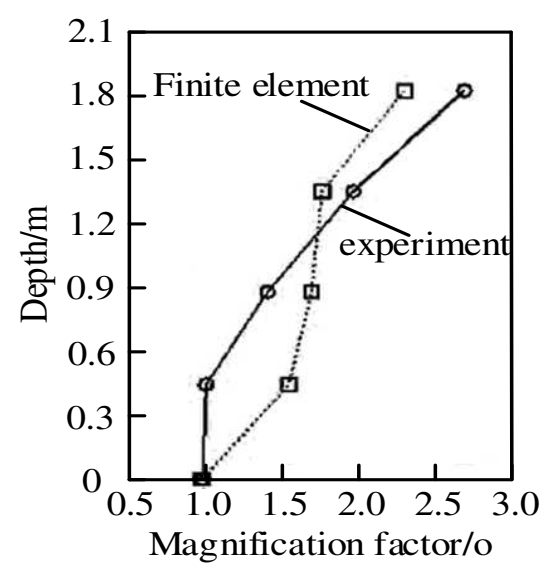


(a) Acceleration amplification factor comparison for the $0.1 \mathrm{~g}$ input

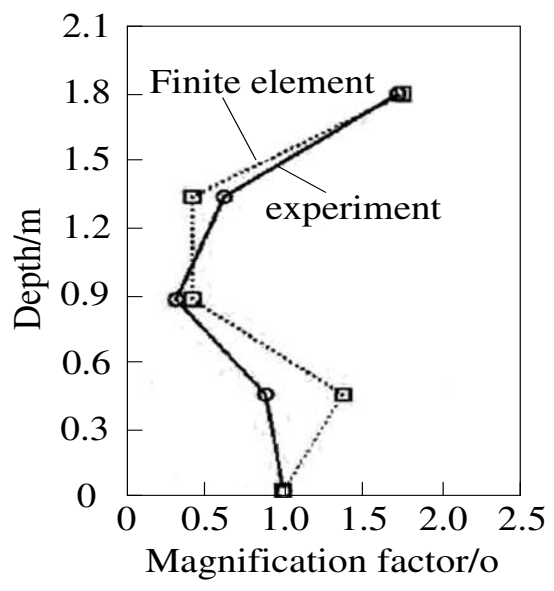

(b) Acceleration amplification factor comparison for the $0.4 \mathrm{~g}$ input

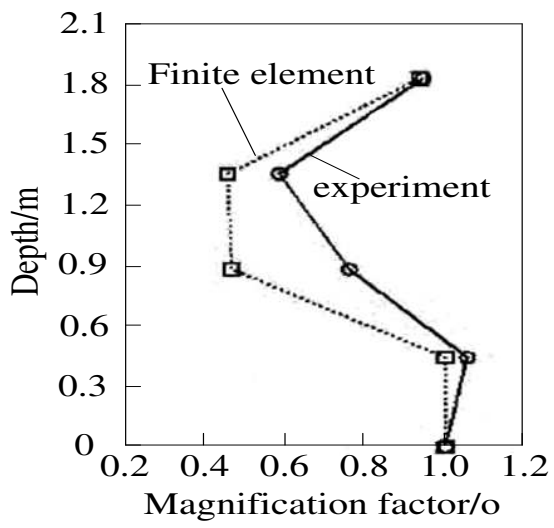

(c) Acceleration amplification factor comparison for the $1.0 \mathrm{~g}$ input

Figure 6 Acceleration amplification factor with different inputs

Figure 6 shows that the finite element calculation results were relatively close to the experimental results, which indicates that the numerical simulation accuracy of the method was relatively high. In the numerical simulation, the accuracy of the model was improved by constructing the joint model of the underground comprehensive pipe gallery.

Figure 7 shows the time history and Fourier amplitude spectrum of the acceleration representative test point AA2 in the lateral non-uniform excitation test of the joints. Other measuring points were similar.
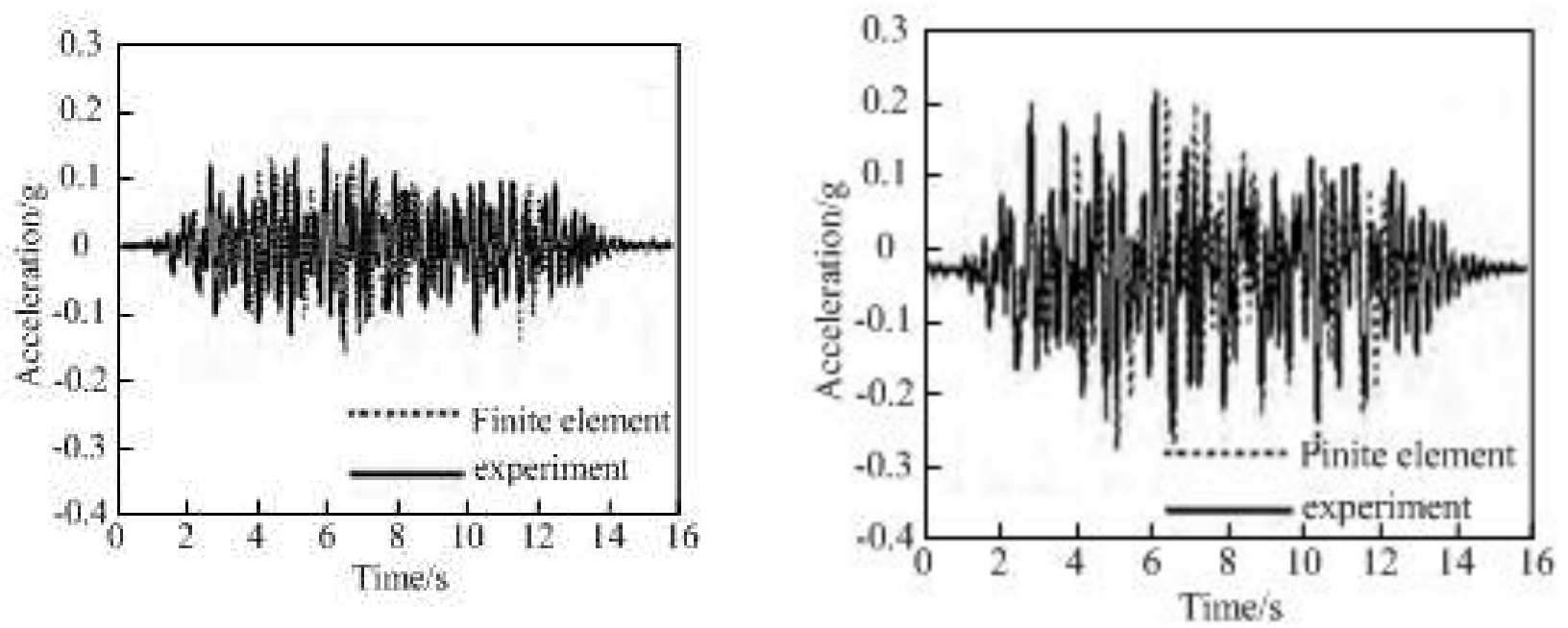
(a) $0.1 \mathrm{~g}$

(b) $0.4 \mathrm{~g}$

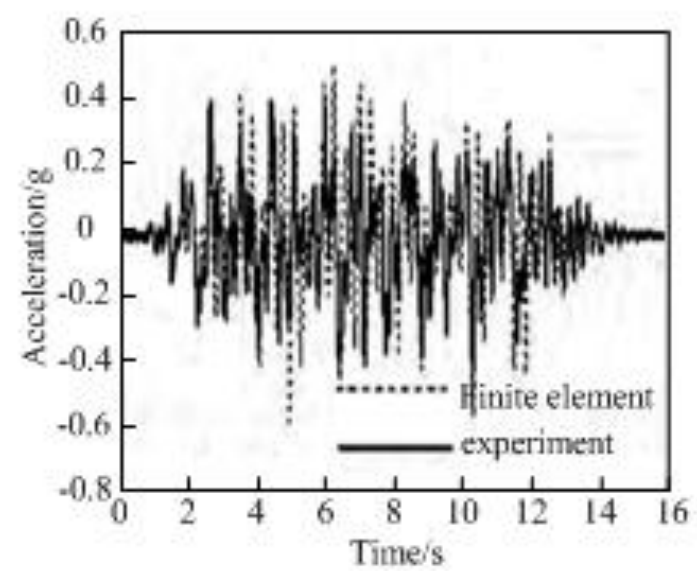

(c) $1.0 \mathrm{~g}$

Figure 7. Acceleration time history of the transverse joint test

Figure 7 shows that the finite element calculation results were close to the experimental results, which indicates that the numerical simulation method for the joint response of the super-large section underground comprehensive pipeline gallery under complex geological conditions based on the finite element model was closer to the analysis of the joint response of the super-large section underground comprehensive pipeline gallery.

The relative displacement of the joint determined by the numerical simulation method for the joint response of the integrated pipe gallery based on the finite element model was compared with the actual displacement, and the results obtained through the experiment are shown in Fig. 8.

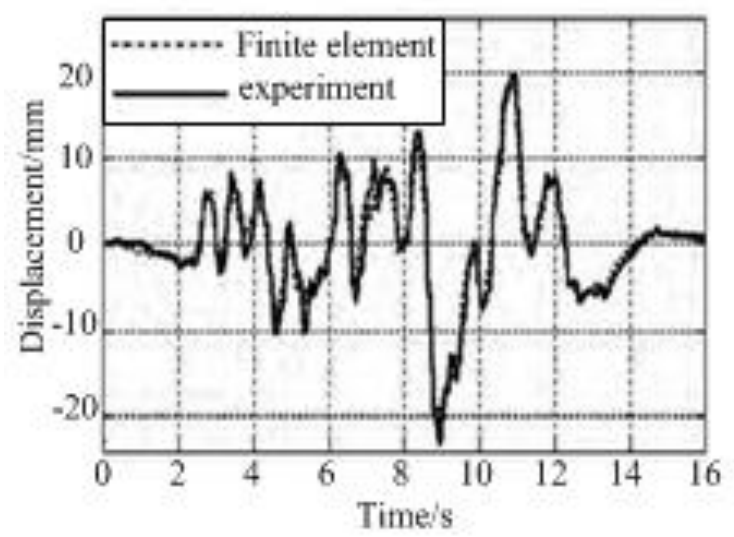

Figure 8. Relative displacement of the joint

Figure 8 shows that the joint displacement determined by the numerical simulation method for the joint response of the integrated pipe gallery based on the finite element model was relatively close to the experimental displacement. It shows that the numerical simulation method for the joint response of the integrated pipe gallery based on the finite element model accurately described the joint displacement of the 
integrated pipe gallery. The accuracy of the numerical simulation method based on the finite element model of the response of the joint response of the super-large section underground comprehensive pipe gallery under the complicated geological conditions was guaranteed.

\section{Conclusion}

The underground integrated pipe gallery, also known as the common ditch, is an underground structure in which two or more lifeline engineering facilities, such as gas, electricity, telecommunications, and water supply and drainage, are laid together. In terms of its structural characteristics, it is different from ordinary pipelines and tunnel structures. The comprehensive pipe gallery generally has a shallow underground structure, and its cross-sectional size is between that of an underground pipeline and a traffic tunnel. Considering the entry of people and the installation of internal facilities, the cross-sectional shape is typically rectangular. Under complex geological conditions, the joints of the underground comprehensive pipeline gallery at the super-large section are prone to damage, which affects the use of the entire pipeline gallery. To alleviate this problem, it is necessary to numerically simulate the interface response of the underground comprehensive pipe gallery at a super-large section under complex geological conditions, and propose a numerical simulation method for the joint response of the super-large section underground comprehensive pipe gallery under complex geological conditions based on the finite element model. The experimental results demonstrated that this method accurately analyzed the joint response of the underground comprehensive pipe gallery, and the accuracy of the numerical simulation was high. This provides a basis for the in-depth research and development of this subject; however, this method still has the problem of a complex modeling process, which affects the speed of the numerical simulation, and relevant experts and scholars need to study and improve the method. With an increasing emphasis on the reduction of urban infrastructure, research on this subject will continue to deepen.

\section{References}

1. Bai, L. et al. Robust scheduling for wind integrated energy systems considering gas pipeline and power transmission n-1 contingencies. IEEE Transactions on Power Systems. 99, 1-1. (2017).

2. Sahu, P., Panigrahi, D. C. \& Mishra. D. P. A comprehensive review on sources of radon and factors affecting radon concentration in underground uranium mines. Environmental Earth Sciences. 7, 617. (2016).

3. Glot, I. et al. Analysis of wave processes in an underground gas pipeline (mathematical model and field experiment). Engineering Failure Analysis. (2021). 
4. Licata, M. et al. Depicting corrosion-born defects in pipelines with combined neutron $/ \gamma$ ray backscatter: a biomimetic approach. Scientific Reports, 10, 1. (2020).

5. Xu, R. H., Jiang R. N. \& Qu T. J. Review of dynamic response of buried pipelines. Journal of Pipeline Systems Engineering and Practice. 12, 2. (2021).

6. Girgin, S. \& Krausmann, E. Lessons Learned from oil pipeline natech accidents and recommendations for natech scenario development - final report. 1, 104. (2015).

7. Botros, K. K. \& Hardeveld, T. V. Pulsation and vibration analysis of compression and pumping systems, in: Pipeline pumping and compression system: a practical approach. ASME Press, (2018).

8. Tian, J. et al. The vibration analysis model of pipeline under the action of gas pressure pulsation coupling, Eng. Fail. Anal. 66, 328-340. (2016).

9. Li, Q. et al., Nonlinear parametric vibration of a 5uid-conveying pipe 5exibly restrained at the ends, Acta Mech. Solida Sin. 33, 327-346. (2020).

10. Bochkarev, N. N. \& Kurochkin, A. A. Vibrodiagnostic motion control of intratubal objects in gas pipeline, Oil and Gas Business. 5, 86-98. (2012).

11. Stajanca, P. et al. Detection of leak-induced pipeline vibrations using fiber-optic distributed acoustic sensing, Sensors, 18, 9: 2841. (2018).

12. Zahab, S. E., Mosleh, F. \& Zayed, T. An accelerometer-based real-time monitoring and leak detection system for pressurized water pipelines, Pipelines. (2016).

13. Lin, G. \& Zhou, M. Vibration detection of spanning subsea pipelines by using a spherical detector, IEEE Access, 7, 7001. (2019).

14. Jia, Z. et al. Experimental study of pipeline leak detection based on hoop strain measurement. Struct. Control Health Monit. 22, 799-812. (2015).

15. K. Lim, L. Wong, W. K. Chiu, \& J. Kodikara. Distributed fiber optic sensors for monitoring pressure and stiffness changes in out-of-round pipes, Struct. Control Health Monit. 23, 303-314. (2016).

16. Bernini, R., Minardo, A. \& Zeni, L. Vectorial dislocation monitoring of pipelines by use of Brillouin-based fiber-optics sensors, Smart Mater. Struct. 17, 1: 015006. (2007).

17. A.B. Lukonge \& X. Cao. Leak detection system for long-distance onshore and offshore gas pipeline using acoustic emission technology, A review. Trans. Indian. Inst. Met. 73, 1715-1727. (2020).

18. Wei, L.et al. Gas pipeline leakage detection based on acoustic technology. Engineering Failure Analysis 31, 1-7. (2013).

19. Mirchev, Y. N. et al. Automatic systems for ultrasonic inspection of pipelines (survey), NDT Days I, 1, 27-37. (2018).

20. Chen, H. et al. Joint spectrum sensing and resource allocation scheme in cognitive radio networks with spectrum sensing data falsification attack. IEEE Transactions on Vehicular Technology. 11, 9181-9191. (2016).

21. Chen, C. K. et al. Analysis of dynamic response of utility tunnel subjected to natural gas explosion loads. Journal of Railway Science and Engineering. 9, 1907-1914. (2017).

22. Shi, Y. Z. et al. Numerical analysis of the influence of soil-structure interface parameters on the seismic dynamic response of underground utility tunnels. China Earthquake Engineering Journal. 5, 811-819. (2017).

23. Zhao, Y. C., Zhu, G. Q. \& Gao, Y. J. Research on temperature field of fire smoke in urban underground utility tunnel in urban underground utility tunnel. Fire Science and Technology. 1, 37-40. (2017).

24. Cao, S. Q. et al. Design and simulation of the automatic process of feed delivery in aquaculture. Computer Simulation. 5, 247-252. (2017). 
25. Fang, Y. C. et al. CFD simulation based on the dynamic response of the vane[J]. Computer Simulation. 37, 2:106-109,158. (2020).

26. Tang, F. \& Li. J. Joint rate adaptation, channel assignment and routing to maximize social welfare in multi-hop cognitive radio networks. IEEE Transactions on Wireless Communications, 4, 2097-2110. (2017). 